\title{
Ex vivo activity of cytotoxic drugs and targeted agents in small intestinal neuroendocrine tumors
}

\author{
Kosmas Daskalakis', Olov Norlén', Andreas Karakatsanis', Per Hellman', Rolf Larsson², \\ Peter Nygren ${ }^{3}$ and Peter Stålberg ${ }^{1}$
}

1Department of Surgical Sciences, Uppsala University, Uppsala, Sweden

2Department of Medical Sciences, Uppsala University, Uppsala, Sweden

3Department of Immunology, Genetics and Pathology, Uppsala University, Uppsala, Sweden

Correspondence should be addressed to K Daskalakis: kosmas.daskalakis@surgsci.uu.se

\begin{abstract}
Small intestinal neuroendocrine tumors (SI-NETs) are generally considered resistant to systemic treatment. To date, predictive markers for drug activity are lacking. Tumor samples from 27 patients with SI-NETs were analyzed ex vivo for sensitivity to a panel of cytotoxic drugs and targeted agents using a short-term total cell kill assay. Samples of renal cancer, colorectal cancer (CRC), ovarian cancer and chronic lymphocytic leukemia (CLL) were included for comparison. For the SI-NET subset, drug sensitivity was analyzed in relation to clinicopathological variables and pre-treatment biomarkers. For cytotoxic drugs, SI-NETs demonstrated similar or higher sensitivity to 5-FU, platinum, gemcitabine and doxorubicin compared with CRC. For several of the targeted kinase inhibitors, SI-NET was among the most sensitive solid tumor types. CLL and ovarian cancer were generally the most sensitive tumor types to both cytotoxic drugs and protein kinase inhibitors. SI-NET was more sensitive to the mTOR inhibitor sirolimus than the other solid tumor types tested. Individual SI-NET samples demonstrated great variability in ex vivo sensitivity for most drugs. Cross-resistance between different drugs also varied considerably, being higher among protein kinase inhibitors. Age, stage, grade, peritoneal carcinomatosis and extra-abdominal metastases as well as serum chromogranin A and urine 5-HIAA concentrations at diagnosis did not correlate to drug sensitivity ex vivo. SI-NETs exhibit intermediate sensitivity ex vivo to cytotoxic and targeted drugs. Clinicopathological factors and currently used biomarkers are not clearly associated to ex vivo sensitivity, challenging these criteria for treatment decisions in SI-NET. The great variability in drug sensitivity calls for individualized selection of therapy.
\end{abstract}

\author{
Key Words \\ - ex vivo activity \\ - cytotoxic drugs \\ - targeted agents \\ - small intestinal \\ neuroendocrine tumor
}

Endocrine-Related Cancer (2018) 25, 471-480

\section{Introduction}

Small intestinal neuroendocrine tumors (SI-NETs) are indolent, low-proliferative and well-differentiated neoplasms. They are often diagnosed at a late stage and have then a 5-year survival rate of $58 \%$ (Norlen et al.
2012). However, variable outcomes are hidden within this relative favorable prognosis.

While locoregional surgery followed by active surveillance remains the mainstay approach for the 
treatment of localized SI-NETs, the management of patients with metastatic disease involves a multimodality treatment approach, combining targeted and systemic approaches with the aims to control hormone production and tumor progression (Niederle et al. 2016).

In contrast to pancreatic NETs and neuroendocrine carcinomas (NECs), SI-NETs are generally considered resistant to systemic cytotoxic drugs with objective response rates in the range of 10-30\% (Sun et al. 2005, Faure et al. 2017). Low proliferation in SI-NETs as well as overexpression of the DNA repair enzyme methylguanine-methyltransferase (MGMT) may contribute to chemotherapy resistance (Kulke et al. 2009).

Total cell kill assays that measure early cell damage in the tumor cell population correlate well with clinical treatment outcomes and are technically robust with high evaluability rates (Bosanquet 1991, Blom et al. 2017). Sensitivity of approximately $90 \%$ and specificity of $70 \%$ for predicting response and resistance to treatment has been reported (Larsson \& Nygren 1993, Blom et al. 2016), indicating that such assays can be used to investigate clinically relevant tumor cell drug sensitivity.

Overall, the experimental and clinical experience on sensitivity of SI-NET to various cancer drugs is limited, leaving a poor basis for treatment decision making in the clinic for advanced SI-NET. The aims of this study were, therefore, to assess sensitivity to a broad panel of cytotoxic drugs and more recently introduced targeted drugs in SI-NETs and to investigate whether established prognostic factors for survival and currently used biomarkers for this tumor type are associated with drug sensitivity.

\section{Materials and methods}

\section{Tumor sampling and cell preparation}

Tumor tissue sampling in different patients with SI-NET, colorectal cancer (CRC), ovarian cancer and renal cancer was performed intra-operatively during resective surgery for primary or metastatic disease. Sampling sites for SI-NETs were primary tumors as well as regional mesenteric lymph node metastases. Chronic lymphocytic leukemia (CLL) sampling was performed by vein puncture at routine blood sampling. Sampling and data collection were based on patient informed consent as approved by the regional ethical committee in Uppsala (Dnr 2007/237 and Dnr 2011/375/1). Due to shortage of cells, all samples were not tested for all drugs.

Tumor cells from solid tumors were prepared by collagenase digestion (Csoka et al. 1997), whereas CLL cells were collected by Ficoll-Hypaque gradient centrifugation (Pharmacia) (Frost et al. 2003). Cells obtained from solid tumors were mostly single cells or small cell clusters with $\geq 90 \%$ viability and less than $30 \%$ contamination from non-malignant cells, as classified after morphological examinations of May-Grünwald-Giemsa-stained cytocentrifugate preparations.

For subgroup analysis of samples from SI-NETs, data on the presence or absence of peritoneal carcinomatosis and extra-abdominal metastasis, tumor grade, disease stage, age as well as pre-treatment s-CgA and u-5HIAA concentrations were collected from the patient charts. For grading and staging of the SI-NETs included, the WHO 2017 classification system was used (Lloyd et al. 2017).

\section{Drug selection and measurement of drug sensitivity ex vivo}

A panel of cytotoxic drugs, commonly used in solid tumors and also tried in NETs, as well as a broad panel of established and more recently introduced tyrosine kinase inhibitors were tested in the SI-NET samples. For comparison and to obtain clinical references, samples from CRC, ovarian cancer, renal cancer and CLL were tested for the same drugs. Temozolomide, an alkylating pro-drug agent used in NETs (Fine et al. 2013, Crespo et al. 2017), produced very little cytotoxicity ex vivo and is, therefore, not included in the data presentation. Additionally, the mTOR inhibitor sirolimus, precursor to the rapalogue everolimus (Yao et al. 2011, 2016), the multikinase inhibitor sunitinib, approved in pancreatic NETs (Raymond et al. 2011), and the somatostatin analogue (SSA) octreotide, extensively used as first-line treatment in metastatic NETs (Cives \& Strosberg 2015), as well as some other tyrosine kinase inhibitors recently approved for other tumor types were tested.

Specifically, the drug panel tested included the cytotoxic drugs 5-fluorouracil (5-FU), cisplatin, docetaxel, doxorubicin, gemcitabine, irinotecan, mitomycin $\mathrm{C}$ and oxaliplatin as well as the targeted agents critzotinib, dasatinib, erlotinib, gefitinib, imatinib, lapatinib, nintedanib, regorafenib, ruxolitinib, sirolimus, sorafenib, sunitinib, and vemurafenib were tested as well as the SSA octreotide.

The drugs were from commercially available clinical preparations or obtained from Selleck Chemicals LLC. The signal transmission pathways targeted by these drugs are indicated in Supplementary Table 1 (see section on supplementary data given at the end of this article). All the drugs were tested at four 10 -fold dilutions from the 
maximal concentration $(\mu \mathrm{M})$ of 30 for cisplatin, 45 for doxorubicin, ruxolitinib and sirolimus, 90 for critzotinib, dasatinib, docetaxel, erlotinib, gefitinib, gemcitabine, imatinib, lapatinib, mitomycin C, nintedanib, oxaliplatin, regorafenib, sorafenib, sunitinib and vemurafenib and 180 for 5-FU, irinotecan and octreotide.

Irinotecan shows relevant activity ex vivo under conditions of the fluorometric microculture cytotoxicity assay (FMCA) despite being considered as a pro-drug (Jonsson et al. 2000).

384-well microplates (Nunc) were prepared with $5 \mu \mathrm{L}$ drug solution at 10 times the final drug concentration using the pipetting robot BioMek 2000 (Beckman Coulter). Tumor cells from patient samples (5000 cells/well for the solid tumor samples and 40,000 cells per well for CLL) in $45 \mu \mathrm{L}$ were seeded in the drug-prepared 384-well plates using the pipetting robot Precision 2000 (Bio-Tek Instruments) or a Biomek 4000 (BeckmanCoulter). Alternatively, and for most samples, drug was added immediately after cell seeding using the liquid handling system ECHO 550 (Labcyte Inc., Sunnyvale, CA, USA). This allows for fast transfer of volumes $\geq 2.5 \mathrm{~nL}$ from source plates into destination wells. In ECHO experiments, source plates were prepared with appropriate concentrations of drugs in DMSO and stored in the oxygen and moisture free MiniPod system (Roylan Developments Ltd, Surrey, UK) until further use. The method for drug addition does not affect assay results. Three columns without drugs served as controls and one column with medium only served as blank.

The semi-automated FMCA was used to determine drug sensitivity (Lindhagen et al. 2008). FMCA is based on the measurement of fluorescence generated from hydrolysis of fluorescein diacetate (FDA) to fluorescein by cells with intact plasma membranes. The culture plates were incubated at $37^{\circ} \mathrm{C}$ in humidified atmosphere. After 72-h incubation, the culture medium was washed away and $50 \mu \mathrm{L} /$ well of a physiological buffer containing $10 \mu \mathrm{g} / \mathrm{mL}$ of the vital dye fluorescein diacetate (FDA) were added to control, experimental and blank wells. After incubation for $30-45 \mathrm{~min}$ at $37^{\circ} \mathrm{C}$, the fluorescence from each well was read in a Fluostar Omega (BMG Laboratories).

\section{Quality control and quantification of results}

Quality criteria for a successful assay were $\geq 70 \%$ tumor cells in the cell preparation prior to incubation and on the assay day in unexposed control wells, as assessed with trypan blue or toluidine blue-stained cytospin preparations, a fluorescence signal in control cultures of $\geq 5$ times mean blank values, and a coefficient of variation of cell survival in control cultures of $\leq 30 \%$. The results obtained by the viability indicator FDA are presented as survival index (SI), defined as the fluorescence of the test expressed as a percentage of control cultures, with blank values subtracted.

Concentration-response SI data formed the basis for calculation of the $50 \%$ inhibitory concentrations $\left(\mathrm{IC}_{50}\right)$, using a non-linear regression to a standard sigmoidal dose-response model.

For comparisons of ex vivo sensitivity between different cancer diagnoses, statistical inference was calculated with 1-way analysis of variance (ANOVA) with Dunnet's post-test and the SI-NET samples as reference. For comparisons of ex vivo sensitivity between different clinicopathological factors as well as biomarker concentrations within the subset of SI-NETs, statistical significance was calculated with the Mann-Whitney test due to small subgroups with distributions deviating from normal. Data are presented as mean values \pm standard error or medians with range as appropriate for the number of experiments/samples indicated. The level of significance was set to two-sided $P<0.05$. Holm-Bonferroni adjustment was used to counteract the problem of multiple comparisons within the SI-NET subset. The statistics software used throughout was GraphPad Prism, version 7 for Macintosh (GraphPad Software).

\section{Results}

Approximately $85 \%$ of all samples fulfilled the criteria for a successful assay and were subsequently included in this study. Sixteen SI-NET patients (59\%) had not received any systemic treatment prior to tumor sampling, while 11 SI-NET patients (41\%) were on somatostatin analogues (SSA) prior to surgery. For CRC and ovarian cancer, 76\% and $43 \%$ of the samples, respectively, were from patients previously treated with chemotherapy, whereas all samples from renal cancer and CLL were from treatment-naive patients. Demographic and clinical characteristics of the SI-NET samples are detailed in Table 1, whereas Table 2 summarizes number and treatment status of all tumor samples investigated.

\section{Drug sensitivity ex vivo}

Samples from SI-NET had lower $\mathrm{IC}_{50}$ values, i.e. were more sensitive, to cytotoxic drugs compared to renal cancer with the exception of mitomycin $\mathrm{C}$ and irinotecan (Fig. 1). Additionally, for half of the protein kinase inhibitors 
Table 1 Patient characteristics of the SI-NET group of 27 patients.

\begin{tabular}{lc}
\hline Patient characteristics & Number $(n$, except for age $)$ \\
\cline { 1 - 1 } Age - mean \pm S.D. & $62.4 \pm 8.1$ \\
Sex & 16 \\
Male & 11 \\
Female & \\
WHO grade & 13 \\
G1 & 14 \\
G2 & \\
TNM stage & 13 \\
III & 14 \\
IV & 11 \\
PC & 4 \\
EAM & \\
\hline
\end{tabular}

EAM, extra-abdominal metastases; PC, peritoneal carcinomatosis.

studied, IC $_{50}$ values in SI-NETS were lower than in renal cancer (Fig. 2).

SI-NET samples compared to CRC demonstrated lower $\mathrm{IC}_{50}$ values for half of the cytotoxic drugs studied (Fig. 1), and for the majority of protein kinase inhibitors studied (7/12; Fig. 2). The CLL and ovarian cancer samples demonstrated $\mathrm{IC}_{50}$ values similar to or lower than the SI-NETs to both cytotoxic drugs and protein kinase inhibitors (Figs 1 and 2). Of notice, SI-NET samples were relatively sensitive to 5-FU, gemcitabine, gefitinib, nintedanib, ruxolitinib and sirolimus whereas irinotecan and docetaxel are not expected to be active in SI-NET. Of particular interest in SI-NET, sirolimus was relatively active, with marginal statistical significance $(P=0.054)$ in comparison with CRC (Fig. 2).

SI-NET samples demonstrated great variability in drug sensitivity with some samples essentially unaffected by the highest drug concentrations tested, whereas other samples exhibited decreased viability even at the lowest concentration (Supplementary Figs 1 and 2). Octreotide demonstrated very low activity (Supplementary Fig. 2).

Cross-resistance between different drugs investigated, varied greatly, being high between protein kinase inhibitors mechanistically related and considerably lower

Table 2 Number of patient samples included in the analyses.

\begin{tabular}{lcc}
\hline Cell type & Patients $(n)$ \\
\cline { 1 - 1 } SI-NET & 27 \\
CRC & 154 \\
Ovarian cancer & 90 \\
Renal cancer & & 54 \\
CLL & 16 \\
\hline
\end{tabular}

\begin{tabular}{c} 
\% previously treated \\
\hline 41 (SSA only) \\
76 \\
43 \\
0 \\
0
\end{tabular}

CLL, chronic lymphocytic leukemia; CRC, colorectal cancer; SSA, somatostatin analogue.

http://ercendocrinology-journals.org https://doi.org/10.1530/ERC-17-0404 for cytotoxic drugs, except for 5-FU and gemcitabine (Supplementary Fig. 3).

\section{Drug sensitivity in subset of SI-NETs}

The median $\mathrm{IC}_{50}$ values for each drug according to different clinicopathological categories and biomarker concentrations in the SI-NET subgroup are presented in Table 3. Age, stage and grade at diagnosis as well as the presence of peritoneal carcinomatosis and extraabdominal metastasis were not related to sensitivity to cytotoxic drugs or the protein kinase inhibitors tested. Likewise s-CgA and u-5HIAA concentrations at diagnosis did not consistently correlate to drug sensitivity.

\section{Discussion}

Treatment of metastatic SI-NETs does not routinely involve cytotoxic drugs and/or systemic targeted agents as a first line of therapy, as such treatment is generally considered to have limited activity in this tumor type (Sun et al. 2005, Kunz et al. 2013). Additionally, symptoms from the secretion of vasoactive mediators from the tumor are often well controlled with SSA and the tumor itself may have an indolent course. However, some welldifferentiated SI-NETs may differ in their proliferative activity between the primary tumor and metastasis, thus making start of tumor controlling therapy a higher priority and choice of therapy complicated (Shi et al. 2015). With the exception of peptide receptor radionuclide therapy (PRRT) as a standard second line of treatment for metastatic SI-NETs, the mTOR inhibitor everolimus has emerged as the treatment of choice to achieve tumor control if local treatment strategies fail (Pavel et al. 2011, Yao et al. 2016, Strosberg et al. 2017). Activity of cytotoxic drugs such as 5-FU/capecitabine with or without oxaliplatin, dacarbazine and temozolomide, as well as of kinase inhibitors like sunitinib and sorafenib has also been observed (Bajetta et al. 2007, Hobday et al . 2007, Kulke et al. 2008, Walter et al. 2010, Fine et al. 2013, Crespo et al. 2017). However, the roles of these drugs in the treatment strategy for metastatic SI-NETs are still not well defined.

In this study, SI-NETs were found generally more sensitive to cytotoxic drugs and targeted agents ex vivo compared to renal cancer, but less sensitive compared to ovarian cancer and CLL. Compared to CRC, SI-NETs were found to be more sensitive to protein kinase inhibitors and some cytotoxic drugs, e.g. 5-FU, cisplatin and doxorubicin. This pattern of drug activity would arbitrarily 


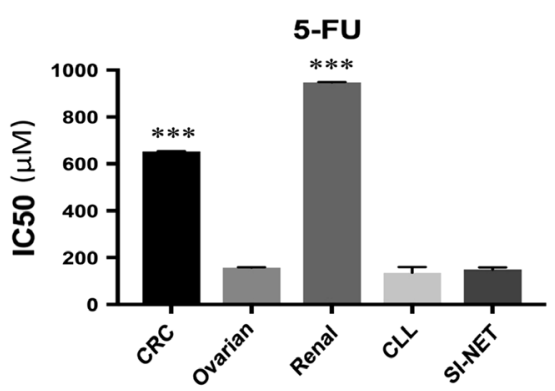

Docetaxel

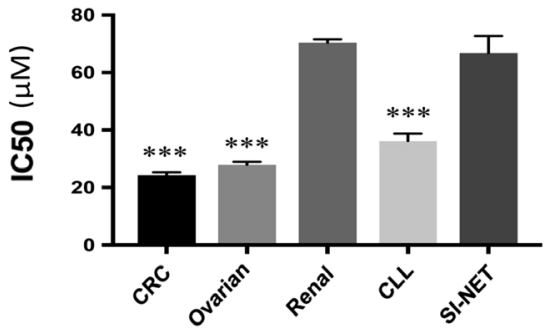

Gemcitabine

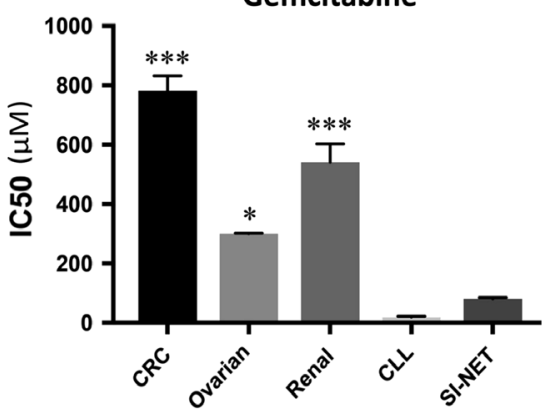

Mitomycin C

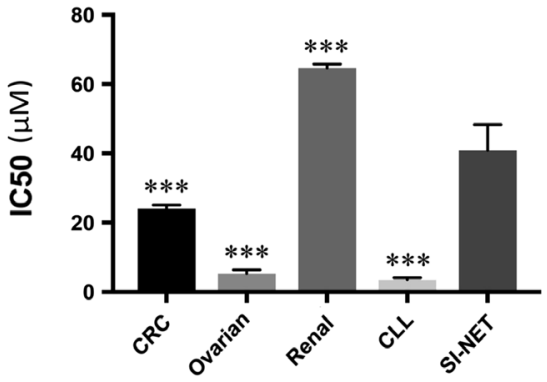

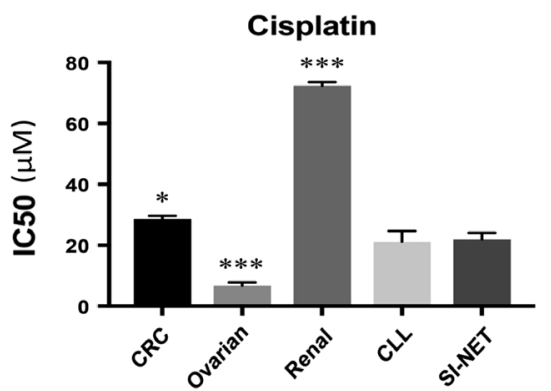

Doxorubicin

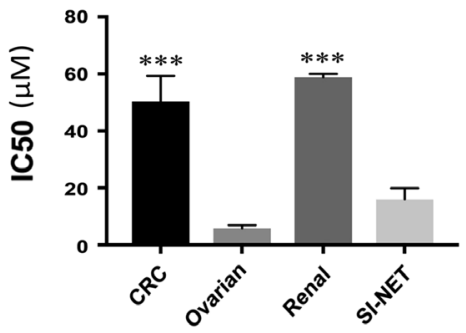

Irinotecan

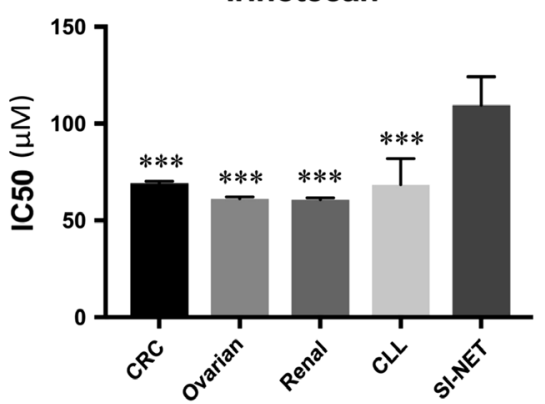

Oxaliplatin

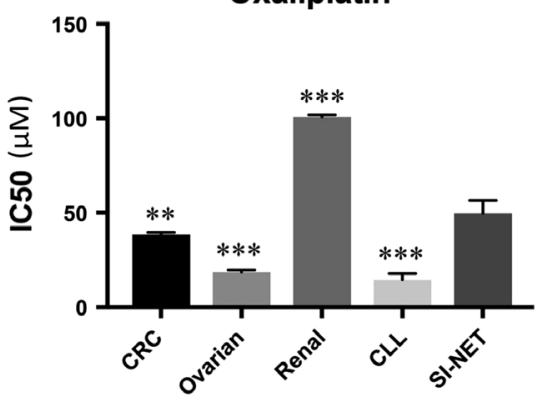

Figure 1

$\mathrm{IC}_{50}$ values for cytotoxic drugs commonly used in solid tumors. Results are presented as means values + S.E. Statistical inference was calculated with 1-way ANOVA with Dunnet's post-test and with the SI-NET samples as reference. * ${ }^{* *}$ and *** denotes $P<0.05,0.001$ and 0.0001 vs SI-NET samples, respectively. Absence of asterisks means that no statistical differences compared to SI-NET samples were observed. label SI-NETs intermediately drug sensitive, similar to e.g. CRC, a tumor type in which several cytotoxic and targeted drugs are now well established to provide benefit in the advanced setting.

Drug activity observed ex vivo using the FMCA might not necessarily transmit to drug activity in vivo, although the FMCA has been shown to adequately reflect the clinical activity of cytotoxic drugs with considerable inter-individual variability in a broad spectrum of other cancer diagnoses (Cashin et al. 2013, Hultman et al. 2014, von Heideman et al. 2014, Bjersand et al. 2015, Blom et al. 2017). However, the tumor microenvironment of SI-NETs might be different than in other cancer types with yet unknown interactions between tumor cells, tumor stroma and/or immune cells that preclude drugs to exert their effects in the patient.

There is to date very scarce or no clinical experience for many of the drugs indicated in the FMCA to be potentially active in SI-NET, notably gemcitabine, gefitinib, nintedanib and ruxolitinib, making it too 

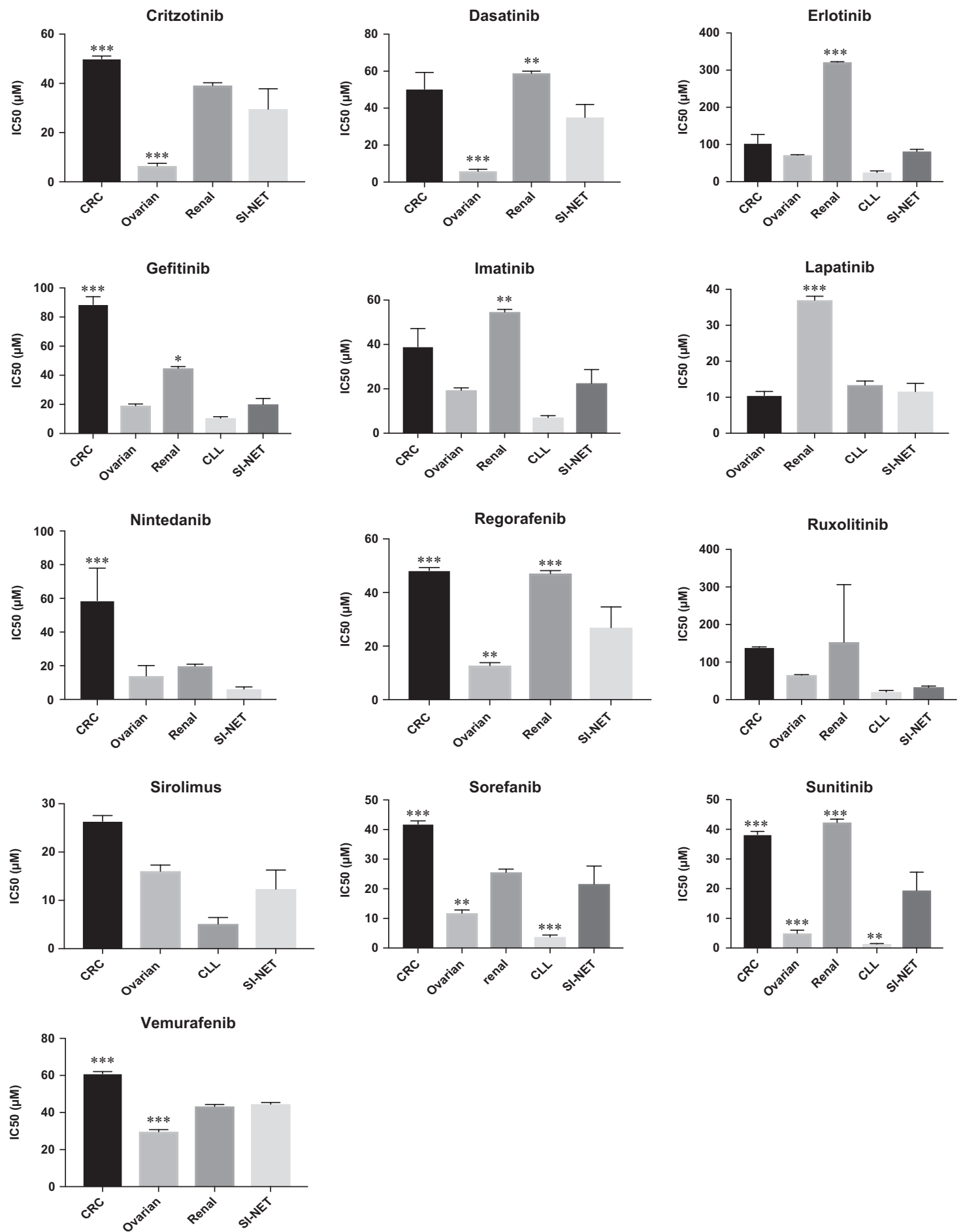

Figure 2

$\mathrm{IC}_{50}$ values for mechanistically differentiated targeted drugs. Results are presented as means values +S.E. Statistical inference was calculated with 1-way ANOVA with Dunnet's post-test and with the SI-NET samples as reference. * ** and *** denotes $P<0.05,0.001$ and 0.0001 vs SI-NET samples, respectively. Absence of asterisks means that no statistical differences compared to SI-NET samples were observed.

early to make conclusions on the ex vivo - clinical drug activity relationship. In this context, it could be noted that drugs that have demonstrated some effect in the clinic, i.e. 5-FU, oxaliplatin and sunitinib were all among drugs being relatively active in the FMCA. Based on this ex vivo assessment of drug activity in SI-NET, especially 


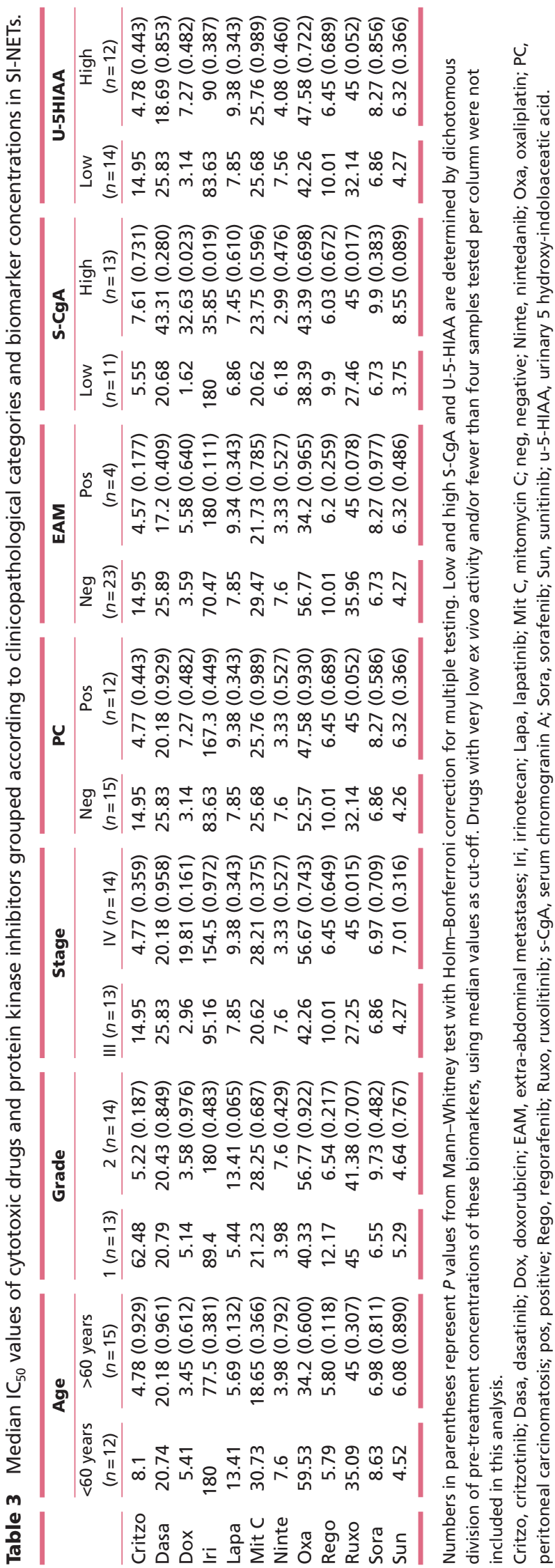

http://erc.endocrinology-journals.org https://doi.org/10.1530/ERC-17-0404
(2) 2018 Society for Endocrinology Published by Bioscientifica Ltd. Printed in Great Britain promising drugs in this tumor type are suggested to be nintedanib and ruxolitinib.

Sirolimus (rapamycin) was only tested in five samples of SI-NET and the $\mathrm{IC}_{50}$ values indicated from these was relatively low compared with the other tumor types, which is also in line with the observed clinical benefit from everolimus in SI-NET (Yao et al. 2016). Importantly, sirolimus is considered to reflect the activity of everolimus (rapamycin analogue).

Apart from anti-secretory activity, somatostatin analogues have demonstrated a stabilizing effect in gastroenteropancreatic-NETs (Rinke et al. 2009, Caplin et al. 2014). This is consistent with the very low ex vivo cytotoxicity of octreotide in this study and in agreement with the direct and indirect mechanisms of somatostatin analogues anti-proliferative action (Cives \& Strosberg 2015).

In the absence of definitive pre-treatment markers as predictive factors for drug response in SI-NET, therapeutic decisions rely currently mostly on clinicopathological criteria. Importantly, the development of novel targeted agents has indeed necessitated the implementation of predictive markers. Mutational status in the PIK3CA/ Akt/mTOR pathway has failed to predict response to everolimus treatment in a clinical setting, probably due to errors related to epigenetic changes (Pavel et al. 2011, Cros et al. 2016, Yao et al. 2016). As protein/mRNA markers in this pathway are lacking, ex vivo drug sensitivity testing may be utilized in order to select patients for treatment with mTOR inhibitors. Regarding other targeted novel therapies, interleukin-8, sVEGFR-3 and SDF-1 $\alpha$ were recently identified as predictors of response to the multiple tyrosine kinase inhibitor sunitinib in a phase II study (Zurita et al. 2015). However, protein kinase inhibitors induce a wide spectrum of changes in the cell, which are not all tested for by available markers, and thus, ex vivo drug sensitivity testing may have a place in individualizing treatment also with protein kinase inhibitors.

Interestingly, in the SI-NETs of this study, clinicopathological factors linked to worse prognosis such as advanced age and stage at diagnosis, tumor grade and the presence of peritoneal carcinomatosis and extra-abdominal metastasis, as well as the currently used biomarkers s-CgA and u-5HIAA, were not clearly associated to ex vivo sensitivity to any kind of drug. These findings indicate that the traditionally used clinicopathological criteria as well as clinically available biomarkers do not reflect tumor cell sensitivity to cytotoxic drugs and targeted agents and are, thus, not immediately predictive for the clinical effect of systemic treatment. 
The cross-resistance between the different cytotoxic drugs and targeted agents investigated varied greatly, with reasonably high correlations between kinase inhibitors known to at least partly share their mechanisms of action (Supplementary Fig. 3 and Supplementary Table 1). In clinical practice, this means that resistance to one of these kinase inhibitors drug would also imply resistance to the other. Nevertheless, individual SI-NET samples could be clearly resistant to one but sensitive to another drug, thus supporting the combined or sequential use of drugs, preferably based on a predictive test like the FMCA. Furthermore, the very conspicuous inter-individual variability between the SI-NET samples in sensitivity to most drugs emphasizes the SI-NET heterogeneity as well as the need for a predictive test.

The strength of this study is the assessment of sensitivity to a broad panel of anti-cancer drugs in a rare tumor type using an assay reported to reflect clinical drug activity. An important limitation of ex vivo drug sensitivity assay used, however, is that only drugs that act directly on the tumor cells can be assayed. Drugs that mainly target stroma or modulate the immune response will require development of novel assays that can account for complex interactions between different cell types (Blom et al. 2017). Furthermore, a considerable fraction of CRC and ovarian cancer samples were from patients previously exposed to chemotherapy. Such samples tend to be somewhat more resistant to drugs ex vivo (von Heideman et al. 2014, Bjersand et al. 2015), implying a certain bias in the comparison between tumor types in this study. Another obvious limitation is the rather low number of tumor samples available for analysis for the SI-NET subset within a reasonably study period, and, thus, few observations on the effect of, e.g. sirolimus and gefitinib. Additionally, FMCA reflects only the pharmacodynamics and not pharmacokinetics of cancer drugs, which explains why this assay is better at predicting in vivo resistance than in vivo activity.

In conclusion, SI-NETs exhibit intermediate, yet variable sensitivity ex vivo to cytotoxic and newly introduced targeted drugs, calling for an individualized choice of therapy in this tumor type. Clinicopathological factors and currently used biomarkers were not associated to ex vivo sensitivity, challenging these criteria in clinical practice. Taken together, in the efforts toward personalized cancer medicine, ex vivo sensitivity testing might have a place for the identification of appropriate cytotoxic drugs and targeted agents for a subset of SI-NETs patients who might benefit from them.
Supplementary data

This is linked to the online version of the paper at https://doi.org/10.1530/ ERC-17-0404.

Declaration of interest

The authors declare that there is no conflict of interest that could be perceived as prejudicing the impartiality of the research reported.

\section{Funding}

The design and conduct of this study as well as collection, management and analysis of the data were supported by the Lions Cancer Foundation, the Göran Gustafsson Foundation for Research in Natural Sciences and Medicine, the Bengt Ihre Research Fellowship, Lennander and Selanders fund and the Swedish Cancer Society.

\section{Author contribution statement}

Drs Nygren and Stålberg contributed equally to this study. Drs Daskalakis, Nygren and Stålberg had full access to all the data in the study and take responsibility for the integrity of the data and the accuracy of the data analysis.

\section{References}

Bajetta E, Catena L, Procopio G, De Dosso S, Bichisao E, Ferrari L, Martinetti A, Platania M, Verzoni E, Formisano B, et al. 2007 Are capecitabine and oxaliplatin (XELOX) suitable treatments for progressing low-grade and high-grade neuroendocrine tumours? Cancer Chemotherapy and Pharmacology 59 637-642. (https://doi. org/10.1007/s00280-006-0306-6)

Bjersand K, Mahteme H, Sundstrom Poromaa I, Andreasson H, Graf W, Larsson R \& Nygren P 2015 Drug sensitivity testing in cytoreductive surgery and intraperitoneal chemotherapy of pseudomyxoma peritonei. Annals of Surgical Oncology 22 (Supplement 3) S810-S816. (https://doi.org/10.1245/s10434-015-4675-0)

Blom K, Nygren P, Alvarsson J, Larsson R \& Andersson CR 2016 Ex vivo assessment of drug activity in patient tumor cells as a basis for tailored cancer therapy. Journal of Laboratory Automation 21 178-187. (https://doi.org/10.1177/2211068215598117)

Blom K, Nygren P, Larsson R \& Andersson CR 2017 Predictive value of ex vivo chemosensitivity assays for individualized cancer chemotherapy: a meta-analysis. SLAS Technology 22 306-314. (https://doi.org/10.1177/2472630316686297)

Bosanquet AG 1991 Correlations between therapeutic response of leukaemias and in-vitro drug-sensitivity assay. Lancet 337 711-714. (https://doi.org/10.1016/0140-6736(91)90287-Y)

Caplin ME, Pavel M, Cwikla JB, Phan AT, Raderer M, Sedlackova E, Cadiot G, Wolin EM, Capdevila J, Wall L, et al. 2014 Lanreotide in metastatic enteropancreatic neuroendocrine tumors. New England Journal of Medicine 371 224-233. (https://doi.org/10.1056/ NEJMoa1316158)

Cashin PH, Mahteme H, Graf W, Karlsson H, Larsson R \& Nygren P 2013 Activity ex vivo of cytotoxic drugs in patient samples of peritoneal carcinomatosis with special focus on colorectal cancer. BMC Cancer 13 435. (https://doi.org/10.1186/1471-2407-13-435)

Cives M \& Strosberg J 2015 The expanding role of somatostatin analogs in gastroenteropancreatic and lung neuroendocrine tumors. Drugs $\mathbf{7 5}$ 847-858. (https://doi.org/10.1007/s40265-015-0397-7)

Crespo G, Jimenez-Fonseca P, Custodio A, Lopez C, Carmona-Bayonas A, Alonso V, Navarro M, Aller J, Sevilla I, Grande E, et al. 2017 
Capecitabine and temozolomide in grade $1 / 2$ neuroendocrine tumors: a Spanish multicenter experience. Future Oncology 13 615-624. (https://doi.org/10.2217/fon-2016-0434)

Cros J, Moati E, Raffenne J, Hentic O, Svrcek M, de Mestier L, Sbidian E, Guedj N, Bedossa P, Paradis V, et al. 2016 Gly388Arg FGFR4 polymorphism is not predictive of everolimus efficacy in well-differentiated digestive neuroendocrine tumors. Neuroendocrinology 103 495-499. (https://doi. org/10.1159/000440724)

Csoka K, Tholander B, Gerdin E, de la Torre M, Larsson R \& Nygren P 1997 In vitro determination of cytotoxic drug response in ovarian carcinoma using the fluorometric microculture cytotoxicity assay (FMCA). International Journal of Cancer 72 1008-1012. (https://doi. org/10.1002/(SICI)1097-0215(19970917)72:6<1008::AIDIJC15>3.0.CO;2-0)

Faure M, Niccoli P, Autret A, Cavaglione G, Mineur L \& Raoul JL 2017 Systemic chemotherapy with FOLFOX in metastatic grade $1 / 2$ neuroendocrine cancer. Molecular and Clinical Oncology 6 44-48. (https://doi.org/10.3892/mco.2016.1097)

Fine RL, Gulati AP, Krantz BA, Moss RA, Schreibman S, Tsushima DA, Mowatt KB, Dinnen RD, Mao Y, Stevens PD, et al. 2013 Capecitabine and temozolomide (CAPTEM) for metastatic, welldifferentiated neuroendocrine cancers: the pancreas center at Columbia University experience. Cancer Chemotherapy and Pharmacology 71 663-670. (https://doi.org/10.1007/s00280-0122055-z)

Frost BM, Nygren P, Gustafsson G, Forestier E, Jonsson OG, Kanerva J, Nygaard R, Schmiegelow K, Larsson R, Lonnerholm G, et al. 2003 Increased in vitro cellular drug resistance is related to poor outcome in high-risk childhood acute lymphoblastic leukaemia. British Journal of Haematology 122 376-385. (https://doi. org/10.1046/j.1365-2141.2003.04442.x)

Hobday TJ RJ, Holen K, Picus J, Donehower RC, Marschke R, Maples W, Lloyd R, Mahoney M \& Erlichman C 2007 MCO44h, a phase II trial of sorafenib in patients (pts) with metastatic neuroendocrine tumors (NET): a phase II consortium (P2C) study. Journal of Clinical Oncology 25 (18 Suppl) 4504.

Hultman B, Mahteme H, Sundbom M, Ljungman M, Larsson R \& Nygren P 2014 Benchmarking of gastric cancer sensitivity to anticancer drugs ex vivo as a basis for drug selection in systemic and intraperitoneal therapy. Journal of Experimental and Clinical Cancer Research 33 110. (https://doi.org/10.1186/s13046-014-0110-9)

Jonsson E, Dhar S, Jonsson B, Nygren P, Graf W \& Larsson R 2000 Differential activity of topotecan, irinotecan and SN-38 in fresh human tumour cells but not in cell lines. European Journal of Cancer 36 2120-2127. (https://doi.org/10.1016/S0959-8049(00)00289-6)

Kulke MH, Lenz HJ, Meropol NJ, Posey J, Ryan DP, Picus J, Bergsland E, Stuart K, Tye L, Huang X, et al. 2008 Activity of sunitinib in patients with advanced neuroendocrine tumors. Journal of Clinical Oncology 26 3403-3410. (https://doi.org/10.1200/ JCO.2007.15.9020)

Kulke MH, Hornick JL, Frauenhoffer C, Hooshmand S, Ryan DP, Enzinger PC, Meyerhardt JA, Clark JW, Stuart K, Fuchs CS, et al. 2009 O6-methylguanine DNA methyltransferase deficiency and response to temozolomide-based therapy in patients with neuroendocrine tumors. Clinical Cancer Research 15 338-345. (https://doi.org/10.1158/1078-0432.CCR-08-1476)

Kunz PL, Reidy-Lagunes D, Anthony LB, Bertino EM, Brendtro K, Chan JA, Chen H, Jensen RT, Kim MK, Klimstra DS, et al. 2013 Consensus guidelines for the management and treatment of neuroendocrine tumors. Pancreas 42 557-577. (https://doi. org/10.1097/MPA.0b013e31828e34a4)

Larsson R \& Nygren P 1993 Laboratory prediction of clinical chemotherapeutic drug resistance: a working model exemplified by acute leukaemia. European Journal of Cancer 29A 1208-1212. (https:// doi.org/10.1016/S0959-8049(05)80316-8)
Lindhagen E, Nygren P \& Larsson R 2008 The fluorometric microculture cytotoxicity assay. Nature Protocols 3 1364-1369. (https://doi. org/10.1038/nprot.2008.114)

Lloyd RV, Osamura RY, Klöppel G, Rosai J, Bosman FT, Jaffe ES, Lakhani SR, Ohgaki H, World Health Organization, International Agency for Research on Cancer, et al. 2017 WHO Classification of Tumours of Endocrine Organs. Lyon, France: IARC Press.

Niederle B, Pape UF, Costa F, Gross D, Kelestimur F, Knigge U, Oberg K, Pavel M, Perren A, Toumpanakis C, et al. 2016 ENETS consensus guidelines update for neuroendocrine neoplasms of the jejunum and ileum. Neuroendocrinology 103 125-138. (https://doi. org/10.1159/000443170)

Norlen O, Stalberg P, Oberg K, Eriksson J, Hedberg J, Hessman O, Janson ET, Hellman P \& Akerstrom G 2012 Long-term results of surgery for small intestinal neuroendocrine tumors at a tertiary referral center. World Journal of Surgery 36 1419-1431. (https://doi. org/10.1007/s00268-011-1296-Z)

Pavel ME, Hainsworth JD, Baudin E, Peeters M, Horsch D, Winkler RE, Klimovsky J, Lebwohl D, Jehl V, Wolin EM, et al. 2011 Everolimus plus octreotide long-acting repeatable for the treatment of advanced neuroendocrine tumours associated with carcinoid syndrome (RADIANT-2): a randomised, placebo-controlled, phase 3 study. Lancet 378 2005-2012. (https://doi.org/10.1016/S01406736(11)61742-X)

Raymond E, Dahan L, Raoul JL, Bang YJ, Borbath I, Lombard-Bohas C, Valle J, Metrakos P, Smith D, Vinik A, et al. 2011 Sunitinib malate for the treatment of pancreatic neuroendocrine tumors. New England Journal of Medicine 364 501-513. (https://doi.org/10.1056/ NEJMoa1003825)

Rinke A, Muller HH, Schade-Brittinger C, Klose KJ, Barth P, Wied M, Mayer C, Aminossadati B, Pape UF, Blaker M, et al. 2009 Placebocontrolled, double-blind, prospective, randomized study on the effect of octreotide LAR in the control of tumor growth in patients with metastatic neuroendocrine midgut tumors: a report from the PROMID Study Group. Journal of Clinical Oncology 27 4656-4663. (https://doi.org/10.1200/JCO.2009.22.8510)

Shi C, Gonzalez RS, Zhao Z, Koyama T, Cornish TC, Hande KR, Walker R, Sandler M, Berlin J, Liu EH, et al. 2015 Liver metastases of small intestine neuroendocrine tumors: Ki-67 heterogeneity and World Health Organization grade discordance with primary tumors. American Journal of Clinical Pathology 143 398-404. (https://doi. org/10.1309/AJCPQ55SKOCYFZHN)

Strosberg J, El-Haddad G, Wolin E, Hendifar A, Yao J, Chasen B, Mittra E, Kunz PL, Kulke MH, Jacene H, et al. 2017 Phase 3 trial of 177Lu-Dotatate for midgut neuroendocrine tumors. New England Journal of Medicine 376 125-135. (https://doi.org/10.1056/ NEJMoa1607427)

Sun W, Lipsitz S, Catalano P, Mailliard JA, Haller DG \& Eastern Cooperative Oncology Group 2005 Phase II/III study of doxorubicin with fluorouracil compared with streptozocin with fluorouracil or dacarbazine in the treatment of advanced carcinoid tumors: Eastern Cooperative Oncology Group Study E1281. Journal of Clinical Oncology 23 4897-4904. (https://doi.org/10.1200/ JCO.2005.03.616)

von Heideman A, Tholander B, Grundmark B, Cajander S, Gerdin E, Holm L, Axelsson A, Rosenberg P, Mahteme H, Daniel E, et al. 2014 Chemotherapeutic drug sensitivity of primary cultures of epithelial ovarian cancer cells from patients in relation to tumour characteristics and therapeutic outcome. Acta Oncologica 53 242-250. (https://doi.org/10.3109/0284186X.2013.794956)

Walter T, Bruneton D, Cassier PA, Hervieu V, Pilleul F, Scoazec JY, Chayvialle JA \& Lombard-Bohas C 2010 Evaluation of the combination 5-fluorouracil, dacarbazine, and epirubicin in patients with advanced well-differentiated neuroendocrine tumors. Clinical Colorectal Cancer 9 248-254. (https://doi.org/10.3816/ CCC.2010.n.037) 
Yao JC, Shah MH, Ito T, Bohas CL, Wolin EM, Van Cutsem E, Hobday TJ Okusaka T, Capdevila J, de Vries EG, et al. 2011 Everolimus for advanced pancreatic neuroendocrine tumors. New England Journal of Medicine 364 514-523. (https://doi.org/10.1056/NEJMoa1009290)

Yao JC, Fazio N, Singh S, Buzzoni R, Carnaghi C, Wolin E, Tomasek J, Raderer M, Lahner H, Voi M, et al. 2016 Everolimus for the treatment of advanced, non-functional neuroendocrine tumours of the lung or gastrointestinal tract (RADIANT-4): a randomised, placebo-controlled, phase 3 study. Lancet 387 968-977. (https://doi org/10.1016/S0140-6736(15)00817-X)

Zurita AJ, Khajavi M, Wu HK, Tye L, Huang X, Kulke MH, Lenz HJ, Meropol NJ, Carley W, DePrimo SE, et al. 2015 Circulating cytokines and monocyte subpopulations as biomarkers of outcome and biological activity in sunitinib-treated patients with advanced neuroendocrine tumours. British Journal of Cancer 112 1199-1205. (https://doi.org/10.1038/bjc.2015.73)

Received in final form 11 January 2018

Accepted 12 February 2018

Accepted Preprint published online 12 February 2018 (c) 2018 Society for Endocrinology Published by Bioscientifica Ltd. 